\title{
O transplante de células-tronco hematopoéticas como opção no tratamento de doenças não hematológicas
}

\section{Hematopoietic stem cell transplant: a therapeutic option for non-hematologic diseases}

Milton A. Ruiz ${ }^{1}$

Lilian Piron- Ruiz ${ }^{2}$

Mario R. Lago

Angelo Maset ${ }^{4}$

Adriana B. Santos 5

Oswaldo T. Greco ${ }^{6}$
Nesta revisão são abordadas as doenças em que existem dados e perspectivas do uso de transplante de células-tronco hematopoéticas em suas diversas modalidades. São apresentados também os aspectos referentes aos regimes de condicionamento empregados, e sua relação com toxicidade e taxa de mortalidade ligadas ao transplante. São apresentadas as doenças autoimunes e particularizados dados especificos do lúpus eritematoso sistêmico, esclerose sistêmica e esclerose múltipla e diabetes mellitus tipo 1. A base do procedimento nas doenças autoimunes é a reprogramação imunológica. Aparentemente o procedimento tem sua indicação nas doenças em que os tratamentos convencionais de imunossupressão tenham falhado, e o dano orgânico não tenha sido definitivo, mas tenha chance de ocorrer caso não seja realizado o transplante. A modalidade aparentemente indicada no momento deve ser o transplante de células-tronco autogênico com regimes de condicionamento não mieloablativo para se obter sobrevivência estimada em mais de $50 \%$ em todas as doenças, com baixa toxicidade e com mortalidade nula ligada a transplante. São apresentados também os resultados nos tumores sólidos, que são discutíveis, e particularidades no câncer de mama. A aparente indicação para os tumores sólidos é transplante de células-tronco alogênico e se baseia no tratamento intensivo com doses mieloablativas com a finalidade de se induzir o efeito enxerto contra o tumor. Os regimes não mieloablativos são preconizados com a finalidade de redução da toxicidade e indução de imunossupressão, sendo os dados insuficientes e discutiveis, o que obriga a introdução de novas estratégias terapêuticas baseadas na terapia imune e celular. Rev. Bras. Hematol. Hemoter. 2009; 31(Supl. 1):68-74.

Palavras-chave: Transplante de células-tronco hematopoéticas; autogênico; alogênico; regimes de condicionamento; doenças autoimunes; tumores sólidos.

\section{Introdução}

O transplante de células-tronco hematopoéticas (TCTH) é uma estratégia terapêutica eficaz no tratamento da anemia aplásica e das doenças onco-hematológicas. ${ }^{1}$ Ele é discutível, controverso ou experimental, nas imunodeficiências, ${ }^{2}$ doenças genéticas e metabólicas, ${ }^{3}$ doenças oncológicas $^{4}$ e nas doenças autoimunes. ${ }^{5-7} \mathrm{Da}$ mesma forma, as modalidades de transplantes autogênica ou alogênica aparentada e não aparentada, a fonte das células para o trans-

${ }^{1}$ Professor colaborador da Disciplina de Hematologia e Hemoterapia da FMUSP-SP e coordenador do Grupo de Terapia Celular do IMC/HMC - São José do Rio Preto-SP

${ }^{2}$ Médica Assistente da Unidade de TCTH, APB-Hospital Infante D. Henrique - São José do Rio Preto-SP.

${ }^{3}$ Biomédico do IMC - São José do Rio Preto-SP.

${ }^{4}$ Médico da APB - Hospital Infante D. Henrique - São José do Rio Preto-SP.

${ }^{5}$ Matemática. Estaticista do Ibilce, São José do Rio Preto - Unesp, SP.

${ }^{6}$ Médico. Chefe do Departamento Científico do IMC, São José do Rio Preto-SP.

Faculdade de Medicina da Universidade de São Paulo - FMUSP-SP

Correspondência: Milton Artur Ruiz

Avenida Dr. Enéas Carvalho de Aguiar, 144 - Cerqueira César

05403-000 - São Paulo-SP - Brasil

E-mail:milruiz@yahoo.com.br

Doi: $10.1590 /$ S1516-84842009005000028 
plante e os regimes de condicionamento têm indicação precisa e determinada nas doenças hematológicas e onco-hematológicas, nas doenças citadas na sequência existe a necessidade de aferir com estudos controlados o papel do TCTH e todas as suas nuances.

Nesta revisão abordaremos as doenças não hematológicas que afetam pacientes em sua maioria na fase adulta, nas quais o TCTH pode ser cogitado como uma opção de tratamento. Os tumores de células germinativas, doenças metabólicas ou erros inatos do metabolismo não serão abordados, mas serão incluídas tabelas com aspectos indicativos da situação destas doenças em relação ao TCTH.

\section{Fontes de informações}

Este trabalho foi baseado em uma pesquisa por meios eletrônicos no Medline, Science Citation Index, Food and Drug Administration (www.fda.gov) e na web site do National Institute of Health (www.clinicaltrials.gov) e no mecanismo de busca do site da Revista Brasileira de Hematologia e Hemoterapia (RBHH) e na coleção SciELO Brasil (www. scielo. br/rbhh). Foram incluídos no texto manuscritos que consideramos consagrados independentes do período de publicação.

\section{Método}

Foram pesquisados, no período de janeiro de 2000 a 2009, as imunodeficiências, doenças neoplásicas, tumores sólidos, doenças metabólicas e doenças autoimunes e, em todos estes grupos de moléstias, as doenças específicas mais frequentes sob os termos que se seguem: Stem cell transplantation, Hematopoietic stem cell transplantation, e Peripheral blood stem cell transplantation.

\section{Doenças autoimunes}

As doenças autoimunes são um grupo de moléstias heterogêneas. Em modelos animais de doenças autoimunes (DAI), induzidas por autopeptídeos ou vacinação, o tratamento com o TCTH alogênico ou autogênico teve sucesso em moléstias como lúpus eritematoso sistêmico (LES), artrite reumatóide (AR), diabetes mellitus insulino dependente, glomerulonefrite crônica e determinados tipos de diabetes mellitus não insulino dependente. ${ }^{8}$

O tratamento das DAI com o TCTH está descrito desde a década de $90{ }^{9-12}$ Até o momento, o registro do Grupo Europeu de Transplante de Medula Óssea (EBMT) relata que mais de 1.300 pacientes com DAI isolada já foram submetidos a transplantes. ${ }^{5} \mathrm{O}$ procedimento está ancorado na reprogramação imunológica. ${ }^{8}{ }^{13-15}$ Esta é obtida após uma quimioterapia mielossupressiva e/ou mieloablativa, seguida da reinfusão de $\mathrm{CTH}$ de doadores ou próprias com a finalidade de restaurar a função medular e imunológica. A escolha da modalidade de transplante para as DAI, aparentemente, é o TCTH autogênico. ${ }^{5,16}$ Isto se deve a menor toxicidade imedi- ata e tardia e a uma mortalidade relacionada ao transplante reduzida. O regime de condicionamento sugerido é o não mieloabalativo e esta escolha recai no fato de se tratarem de doenças não malignas, sendo, portanto, imprescindível à baixa toxicidade e mortalidade relacionadas ao transplante. ${ }^{16,17}$ Outro dado adicional é que não existem diferenças significativas em relação à sobrevida livre de sintomas de doenças após cinco anos quando os regimes de condicionamento são comparados. ${ }^{17-20}$ Outro aspecto a ser ressaltado é que os pacientes submetidos ao TCTH alogênico apresentam risco de desenvolverem tardiamente síndromes mielodisplásicas/leucemias, ${ }^{19}$ ou de segunda neoplasia em pacientes submetidos ao TCTH. ${ }^{21,22}$ Sempre é bom lembrar que o objetivo principal do tratamento das DAI é o de modificar a história natural da doença muito mais que a obtenção da cura, não devendo também ser esquecido que existem relatos de melhoras espontâneas ou de estabilidade destas doenças em diversos pacientes. ${ }^{8}$

A indicação do TCTH segundo o EBMT deve seguir os critérios abaixo: ${ }^{5}$

1. A doença deve ser grave, ter risco elevado, estar em fase avançada ou em progressão, com possibilidade de evoluir para comprometimento orgânico irreversível.

2. A doença deve ser refratária aos tratamentos convencionais.

3. O TCTH deve ser realizado antes do estabelecimento de um comprometimento orgânico irreversível, para que o possível benefício clínico do transplante possa ser aferido.

Dentre as doenças tratadas com o TCTH podemos citar: lúpus eritematoso sistêmico (LES), ${ }^{8-10}$ esclerose, sistêmica (ES) ${ }^{23-25}$ artrite reumatóide (AR), ${ }^{26}$ esclerose múltipla (EM) ${ }^{27-29}$ e diabetes mellitus do tipo $1(\mathrm{DM} 1),{ }^{30}$ dentre outras.

No LES refratário, sintomático e com comprometimento orgânico, os resultados com o TCTH autogênico com regime não mieloablativo é semelhante ao de estudo com regime mieloablativo. Em ambos foi observado que a sobrevida livre de doença após cinco anos foi de $50 \%$. No entanto, a taxa de mortalidade ligada ao procedimento entre o TCTH autogênico e o alogênico foi de $2 \%$ para $13 \% .^{31,32}$

Nos estudos da esclerose sistêmica, doença que apresenta o maior número de transplantes no EBMT após a esclerose múltipla (MS), o TCTH autogênico não mieloablativo mostrou também ter uma menor taxa de mortalidade, inferior a $4 \%$, enquanto no regime mieloablativo ela foi de $23 \%$. Em ambos os estudos, após cinco anos foi descrita uma taxa de $64 \%$ de sobrevida livre de eventos. ${ }^{23,24}$

AMS é uma doença inflamatória imune mediada. Após diversos estudos com regimes mieloablativos seguidos de alta toxicidade e de mortalidade relacionada ao transplante, existe hoje o consenso de que o TCTH autogênico com regimes não mieloabalativos deve ser a praxe, pois o mesmo reverte a disabilidade enquanto a mortalidade relacionada ao transplante está ao redor de zero. ${ }^{29,33}$ Nesta última déca- 
da, o TCTH autogênico tem sido explorado na MS em seus vários aspectos, quais sejam factibilidade, toxicidade, impacto na progressão da doença, achados de Ressonância Magnética e a utilização de mobilizações e associações medicamentosas. Iremos nos ater a dados sólidos, publicados pelo Grupo de Trabalho de Doenças AutoImunes da Sociedade Européia de Transplantes de Medula Óssea que iniciou a coleta de informações através de um banco de dados organizado em 1997. Já houve 2 comunicações. 28,33 Na segunda comunicação, ocorrida em 2006, Saccardi e col ${ }^{33}$ relataram a experiência acumulada em 183 pacientes que se submeteram ao TCTH autogênico. As taxas de mortalidade foram de 5,3\%, e se restringiram ao período anterior ao ano de 2000, relacionadas a regimes terapêuticos distintos. Também existiu correlação entre toxicidade, técnicas de transplante, e condições clínicas no momento do transplante. A melhora ou estabilização do quadro neurológico ocorreu em $63 \%$ dos pacientes, com um seguimento médio de 41,7 meses. Assim, aquele grupo de trabalho concluiu que o TCTH autogênico em esclerose múltipla era um procedimento promissor e que diminuia a progressão da doença em pacientes acometidos de MS severa e progressiva, e um estudo fase III foi iniciado. Obviamente, a segurança e aplicabilidade do TCTH autogênico dependem da correta seleção de pacientes e associações medicamentosas. Outras moléstias que apresentam resultados alvissareiros são as doenças de Chrön, polineuropatia inflamatória desmielinizante crônica, dermatomiosite/poliomiosite, poliarterite nodosa, doença de Behcet, arterite de Takayasu e diabetes mellitus tipo $1.8,30,34,35$ Em relação a esta doença, recentes relatos demonstram que a precocidade do TCTH com regime não mieloablativo propicia remissão do quadro desta doença com suspensão do uso de insulina na maioria dos pacientes (14/15) com baixa toxicidade e ausência de mortalidade relacionada ao transplante. ${ }^{35}$

Apesar dos resultados serem promissores, existem dúvidas que ainda persistem em relação ao TCTH. Isto pode ser observado na plataforma de registro de estudos clínicos americana. Um grande número de DAI encontra-se sob estudo (46 registros), sendo que mais da metade destes abordam especificamente o lúpus e a esclerose múltipla.

Observa-se também uma grande preocupação em relação à segurança do procedimento, e em vários deles a abordagem é do TCTH versus altas doses de imunossupressão, com o emprego de anticorpos monoclonais com a finalidade de se obter a depleção de células T.36,37,38
A impressão final é que, apesar dos resultados promissores em diversas doenças, o papel definitivo do TCTH e de suas várias nuances, como já dito anteriormente, ainda está por ser definido.

Na sequência, como exposto, apresentamos várias tabelas. Na Tabela 1 estão as imunodeficiências mais comumente tratadas com TCTH; na Tabela 2 apresentamos a situação atual frente ao TCTH nas doenças metabólicas; e nas Tabelas 3 e 4 algumas doenças hematológicas menos frequentemente transplantadas e a sua situação atual. ${ }^{3}$

Tabela 1. Imunodeficiências tratadas com $\mathrm{TCTH}^{3}$

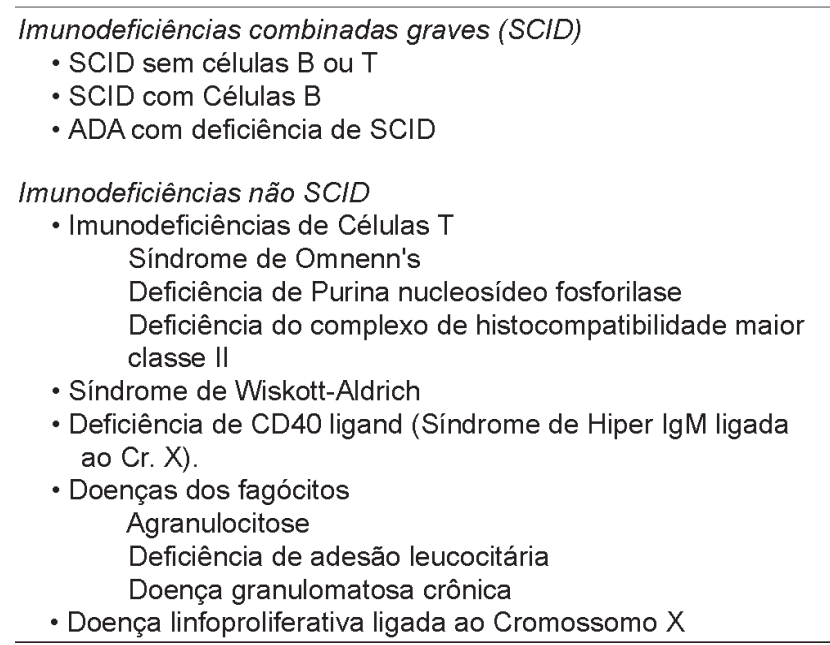

Tabela 2. Doenças metabólicas e situação atual frente ao $\mathrm{TCTH}^{3}$

\begin{tabular}{ll}
\hline \multicolumn{1}{c}{ Doenças } & \multicolumn{1}{c}{ Indicações } \\
\hline Adrenoleucodistrofia & Indicado em pacientes selecionados \\
Aspartylglicosaminuria & Estudos pilotos \\
Doença de Batten & Estudos pilotos \\
Fucosidosis & Estudos pilotos \\
Doença de Gaucher tipo I e III & Estudos pilotos \\
Leucodistrofia de Células globoides (GLD) & \\
$\quad$ Doença de Krabbe & Estudos pilotos \\
GLD de início tardio & Protocolos clínicos sujeitos a aprovação \\
Alfa Mannosidosis & Protocolos clínicos sujeitos a aprovação \\
Leucodistrofia metacromática (MLD) & \\
MLD tardia da infância & Estudos pilotos \\
MLD juvenil ou do adulto & Protocolos clínicos sujeitos a aprovação \\
Mucolipidosis tipo II & Estudos pilotos \\
Mucopolisacaridoses (MPS) & \\
Síndrome de Hurler & Indicado em pacientes selecionados \\
MPS tipo IV & Protocolos clínicos sujeitos a aprovação \\
MPS tipo VII & Protocolos clínicos sujeitos a aprovação \\
MPS tipo II, II e IV & Contraindicado \\
GM1 e GM2 gangliosidosis & Contraindicado \\
Doença de Newmann-Pick tipo A e C & Contraindicado \\
\hline
\end{tabular}




\begin{tabular}{l} 
Tabela 3. Doenças hematológicas raras tratadas com o TCTH \\
\hline Insuficiência medular e citopenias \\
- Pancitopenia \\
Anemia de Fanconi \\
Sindrome de Schwachman-Diamond \\
Disqueratose congênita \\
- Aplasia de série vermelha \\
Síndrome de Blackfan-Diamond \\
- Neutropenia \\
Neutropenia congênita grave \\
- Trombocitopenia \\
- Trombocitopenia amegacariocítica congênita \\
Doenças fagociticas com comprometimento orgânico \\
- Síndromes Hemofagocíticos T \\
Linfohistiocitose hemofagocítica \\
Síndrome de Chediak-Higashie \\
Síndrome de Griscelli tipo 2 (Mutação RAB27A) \\
- Osteopetrose maligna da infância
\end{tabular}

governamentais que haviam desestimulados novos estudos em decorrência dos resultados sul-africanos e propunham restrito seguimento em relação ao código de ética para condução de novos estudos para o câncer de mama. ${ }^{45}$

Os tumores sólidos em geral continuam a utilizar o TCTH como uma forma de tratamento. ${ }^{46}$ Isto pode ser aferido na Tabela 5, onde podemos observar que, no ano de 2006, na Europa foram realizados 1.564 transplantes (85 Alo e 1.479 Auto). A doença que mais se transplantou foi o neuroblastoma seguida do câncer de mama. ${ }^{47}$

Em 66 pacientes de alto risco com câncer de mama metastático de 15 centros, foi avaliada a eficácia do TCTH alogênico. Trinta e nove pacientes $(59 \%)$ receberam regime mieloablativo e $27(41 \%)$ regime de intensidade reduzida (RIC). O grupo que recebeu RIC tinha um pior performance status (PS), (63\% vs 23\%), e foi também o que desenvolveu menos doença enxerto versus o hospedeiro $(\mathrm{DECH})$ após um ano (8/36\%). A taxa de mortalidade após cem dias foi menor no grupo RIC (7/29\%), e a sobrevida livre de progressão também foi menor neste grupo após um ano de avaliação (8/23\%). Ficou claro, principalmente, que as mulheres do grupo $\mathrm{RIC}$, que desenvolveram DECH agudo, tiveram menor chance de recaída ou de progressão de moléstia, dado consistente com o desenvolvimento do efeito enxerto contra o tumor. ${ }^{48}$

Outro estudo recente em 28 pacientes com câncer de mama inflamatório de estágio III B foi avaliado em relação ao TCTH autogênico. O transplante foi realizado nos pacientes após altas doses de quimioterapia (thiotepa + mitoxantrone + carboplatina) como regime de condicionamento e a mobilização para coletas de CTH com fatores de crescimento. Alguns pacientes

\section{Tumores sólidos}

O TCTH nos tumores sólidos permanece controverso e discutível. Desde o inicio da década de 90, inúmeros ensaios clínicos foram propostos, principalmente os dirigidos para o tratamento do carcinoma metastático ou inflamatório de mama. ${ }^{39,40,41}$ Em 1995, um artigo sul-africano apresentou resultados impressionantes com altas doses de quimioterápicos seguido do TCTH em relação à sobrevida global e livre de doença. ${ }^{42}$ Estes resultados inibiram outros estudos randomizados que estavam em evolução, e os resultados de outros estudos não confirmaram o estudo sul-africano. Em 1999, o mesmo autor confirmou os resultados em nova publicação. ${ }^{43}$ Após uma auditoria, os estudos foram considerados fraudulentos. ${ }^{44} \mathrm{O}$ comunicado logo se fez sentir com denúncias frente a novos estudos como censura à imprensa leiga e a órgãos que tiveram indicações receberam, após o transplante, radioterapia e tamoxifen. A sobrevida global e a sobrevida livre de progressão de doença avaliadas após 15 anos apresentaram os seguintes resultados: a sobrevida global foi de 49,5 meses e a mediana de sobrevida livre de progressão de doença de 40 meses. Na última avaliação, 16 pacientes haviam recidivado e o procedimento foi considerado seguro, com baixa toxicidade, além de não ter ocorrido nenhum óbito relacionado ao transplante. ${ }^{49}$

Apesar do apresentado, não existem dados suficientes que indiquem como rotina o uso de altas doses de quimioterapia seguido do TCTH. O TCTH alogênico apresenta alta taxa de morbidade, piora a qualidade de vida dos pacientes, assim como pairam dúvidas em relação à melhora significativa após um ano do procedimento, sendo necessário, então, melhor seleção dos pacientes e a realização de estudos clíni- 
Tabela 5. Pacientes submetidos ao TCTH, segundo tipo, doador e fonte de células na Europa no ano de 2006 segundo o EBMT 47

\begin{tabular}{|c|c|c|c|c|c|c|c|c|c|}
\hline $\begin{array}{c}\text { Tipo } \\
\text { Doador } \\
\text { Fonte } \\
\end{array}$ & Neuro & Sar & $\begin{array}{c}\text { Tu } \\
\text { Germ }\end{array}$ & Ca Mama & Ewing & $\begin{array}{c}\mathrm{Ca} \\
\text { Renal }\end{array}$ & Mel & $\begin{array}{c}\text { Ca } \\
\text { Cólon }\end{array}$ & Outros \\
\hline \multicolumn{10}{|l|}{ Alo Ap Id } \\
\hline MO & 7 & 1 & 1 & 8 & 2 & 6 & 2 & & 3 \\
\hline $\mathrm{SP}$ & 1 & 1 & & & 2 & & & & 8 \\
\hline SCU & 2 & & & & & & & & \\
\hline \multicolumn{10}{|l|}{ Alo Ap N Id } \\
\hline MO & 1 & & & & & & & & \\
\hline $\mathrm{SP}$ & 9 & 7 & 1 & 4 & & & & & 1 \\
\hline \multicolumn{10}{|l|}{ SCU } \\
\hline $\begin{array}{l}\text { Alo Ap } \\
\text { gemelar }\end{array}$ & 2 & 2 & & & & & & & \\
\hline \multicolumn{10}{|l|}{ Alo N Ap } \\
\hline MO & 3 & 3 & 1 & & & & & & \\
\hline $\mathrm{SP}$ & & & 1 & 3 & 3 & 2 & & & 1 \\
\hline SCU & & & & & & & & & 1 \\
\hline \multicolumn{10}{|l|}{ Auto } \\
\hline MO & 37 & 9 & 7 & 134 & 9 & 1 & & & 14 \\
\hline $\mathrm{MO}+\mathrm{SP}$ & 295 & 63 & 299 & & 237 & 7 & & 5 & 362 \\
\hline Alo Total & 25 & 10 & 4 & 15 & 7 & 8 & 2 & 0 & 14 \\
\hline Auto Total & 332 & 72 & 306 & 134 & 246 & 8 & & 5 & 376 \\
\hline Total & 357 & 82 & 310 & 149 & 253 & 16 & 2 & 5 & 390 \\
\hline
\end{tabular}

Neuro - Neuroblastoma; Sarc - Sarcoma de tecidos moles; Tu Germ - Tumores germinativos; Ca mama - Carcinoma de mama; Ca renal - Carcinoma renal; Mel - Melanoma; Ca colon - carcinoma de colon ; Alo Ap ld - alogênico aparentado idêntico; Alo Ap N Id - Alogênico não aparentado idêntico; Auto - autogênico; MO - Medula óssea; SP-Sangue periférico; SCU - Sangue de cordão umbilical

cos randomizados para responder a questão da indicação do transplante nos vários tipos e fases do câncer de mama. $\mathrm{Na}$ plataforma de registros de estudos clínicos americana existem 117 estudos de câncer de mama com TCTH.

Em outros tumores sólidos, o TCTH tem sido utilizado e a literatura sempre demonstra casuística pequena e preferência para a modalidade alogênica com a finalidade de erradicação da moléstia, induzir a DECH para se obter um efeito antitumoral. ${ }^{50-54}$ Os resultados atuais são tímidos, com alta toxicidade seguida de morbidade e mortalidade, abrindo perspectivas para novas estratégias baseadas na terapia imune e celular, que podem ser apropriadas ao tratamento de tumores sólidos e a cânceres refratários..$^{55}$

\section{Abstract}

In this report we discuss data and perspectives of hematopoietic stem cell transplantation in non-hematologic diseases. Aspects related to the conditioning regimen and its relationship with toxicity and mortality are also presented. Specific autoimmune diseases are discussed, in particular systemic lupus erythematosus, systemic sclerosis, multiple sclerosis and type 1 diabetes mellitus. The aim of the procedure in autoimmune diseases is immune reprogramming. Apparently this procedure has indications for diseases in which conventional treatments have failed when organ damage is not definitive, but likely to occur if transplantation is not performed. The most promising method appears to be autologous stem cell transplantation with non-myeloablative conditioning regimens to obtain survival that is estimated at more than 50\% for all autoimmune diseases, with low toxicity and no mortality related to transplantation. The controversial results of solid tumor treatment and particularities of breast cancer are also presented. Hematopoietic stem cell transplantation is the apparent indication for solid tumors based on intensive treatment with myeloablative doses in order to induce the graft versus tumor effect. The myeloablative conditioning regimens are introduced with the purpose of reducing the toxicity and inducing immunosuppression but the data are insufficient and questionable requiring the introduction of new therapeutic strategies based on cellular and immune therapy. Rev. Bras. Hematol. Hemoter.

Key words: Hematopoietic stem cell transplantation; autologous; allogeneic; conditioning regimen; autoimmune diseases; solid tumors. 


\section{Referências Bibliográficas}

1. Ljungman $\mathrm{P}$, Urbano-Ispizua A, Caravazzana-Calvo $\mathrm{M}$, et al. Allogeneic and autologous transplantation for haematological diseases, solid tumours, and immune disorders: definitions and curent practice in Europe. Bone Marrow Transplant. 2006;37(5):439-49.

2. Antoine C, Muller S, Cant A. Long term survival and transplantation of haematopoietic stem cells for immunodeficiencies: report of the european experience 1968-1999. Lancet. 2003;361:553-60.

3. Steward CG, Jarisch A. Haemopoietic stem cell transplantation for genetic disorders; Arch Dis Child. 2005;90(12):1259-63.

4. Bregni M. HSCT for solid tumours cap. 31; 464-471 in Apperley J, Carreras E, Gluckman E, Gratwohl A e Masszi T. The EBMT Hand Book - haematopoietic Stem Cell Transplantation - EBMT/ ESH, Forum Service Editore Italia -5a. ed. 2008, 592 p.

5. Saccardi R, Farge D; HSCT for autoimmune diseases in adults cap. 32: 473 in Apperley J, Carreras E, Gluckman E, Gratwohl A. e Masszi T. The EBMT Hand Book - haematopoietic Stem Cell Transplantation - EBMT/ESH, Forum Service Editore Italia -5a. ed. 2008, 592 p.

6. Tyndall A, Saccardi R. Haematological stem cell transplantation in the treatment of severe autoimmune disease: results from phase I/II studies prospective randomized trials and future directions. Clin Exp immunol. 2005;141(1):1-9.

7. Dazzi F, van Laar JM, Cope A, Tyndall A. Cell therapy for autoimmune diseases. Arthritis Res. Ther. 2007;9(2):206-14.

8. Burt RK, Loh Y, Pearce W, Beohar N, Barr WG, et al. Clinical applications of blood-derived and marrow-derived stem cells for nonmalignant diseases JAMA. 2008;299(8):925-93.

9. Marmont AM, van Lint MT, Gualandi F, Bacigalupo A. Autologous marrow stem cell transplantation for severe systemic lupus erythematosus of long duration. Lupus. 1997;6:545-8.

10. Burt RK, Traynor A, Ramsey-Goldman R. Hematopoietic stem cell transplantation for systemic lupus erythematosus. N Eng J. Med. 1997;337:1777-8.

11. Voltarelli JC. Hematopoietic stem cell transplantation for autoimmune diseases in Brazil: current status and future perpectives. Rev. Bras. Hematol. Hemoter. 2002;24(3):206-11.

12. Voltarelli JC. Transplante de células-tronco hematopoéticas para doenças autoimunes no Brasil. Rev. Bras. Hematol. Hemoter. 2002; 24(1):09-13.

13. Sikes M, Nikolic B. Treatment of severe auto immune disease by stem cell transplantation. Nature. 2005;435:620-7.

14. Roux E, Dumont-Girard F, Starobinski M, Siegrist CA, Helg C, et al. Recovery of immune reactivity after T-cell-depleted bone marrow transplantation depends on thymic activity. Blood. 2000;96(6):2299-303.

15. Alexander T, Thiel A, Rosen O, Massenkeil G, Sattler A, et al. Depletion of autoreactive immunologic memory followed by autologous hematopoietic stem cell transplantation in patients with refractory SLE induces long-term remission through de novo generation of a juvenile and tolerant immune system. Blood. 2009; 113(1)214-23.

16. Gratwohl A, Passweg J, Bocelli-Tyndall C, et al. Autoimmune diseases working party of the European Group for Blood and Marrow Transplantation (EBMT). Autologous hematopoietic stem cell transplantation for autoimmune diseases. Bone Marrow Transplant. 2005;35(9):869-79.

17. Nash RA, McSweeney PA, Crofford LJ, et al. High-dose immunosuppressive therapy and autologous hematopoietic cell transplantation for severe systemic sclerosis: long-term followup of the US multicenter pilot study. Blood. 2007;110(4): 1388-96.
18. Binks M, Passweg JR, Furst D, et al. Phase I/II trial of autologous stem cell transplantation in systemic sclerosis: procedure related mortality and impact on skin disease. Ann Rheum Dis. 2001; 60(6):577-84.

19. Burt RK, Marmont A, Oyama Y, et al. Randomized controlled trials of autologous hematopoietic stem cell transplantation for autoimmune diseases: the evolution from myeloablative to lymphoablative transplant regimens. Arthritis Rheum. 2006;54 (12):3750-60.

20. Statkute L, Verda L, Oyama Y, et al. Mobilization, harvesting and selection of peripheral blood stem cells in patients with autoimmune diseases undergoing autologous hematopoietic stem cell transplantation. Bone Marrow Transplant. 2007;39(6):317-329

21. Friedman DL, Rovo A, Leisenring W, Locasciulli A, Flowers ME, et al. Increased risk of breast cancer among survivors of allogeneic hematopoietic cell transplantation: a report from the FHCRC and the EBMT-Late Effect Working Party. Blood. 2008;111(2):939-44.

22. Lowe T, Luu T, Shen J, Bhatia S, Shibata S, et al. Male breast cancer 15 years after allogeneic hematopoietic cell transplantation including total body irradiation for recurrent acute lymphoblastic leukemia. Onkologie. 2008;31(5):266-9.

23. Oyama Y, Barr WG, Statkute L, et al. Autologous non-myeloablative hematopoietic stem cell transplantation in patients with systemic sclerosis. Bone Marrow Transplant. 2007;40(6):549-55.

24. Vonk MC, Marjanovic Z, van den Hoogen FH, et al. Long-term follow-up results after autologous haematopoietic stem cell transplantation for severe systemic sclerosis. Ann Rheum Dis. 2008;67(1):98-104.

25. Farge D, Passweg J, van Laar JM, et al. Autologous stem cell transplantation in the treatment of systemic sclerosis: report from the EBMT/EULAR registry. Ann Rheum Dis. 2004; 63(8): 974-981.

26. Teng YK, Verburg RJ, Sont JK, van den Hout WB, Breedveld FC, van Laar JM. Long-term follow-up of health status in patients with severe rheumatoid arthritis after high-dose chemotherapy followed by autologous hematopoietic stem cell transplantation. Arthritis Rheum. 2005;52(8):2272-6.

27. Kozák T, Havrdová E, Pit'ha J, et al. High-dose immunosuppressive therapy with PBPC support in the treatment of poor risk multiple sclerosis. Bone Marrow Transplant. 2000;25(5):525-31.

28. Fassas A, Passweg JR, Anagnostopoulos A, et al. Autoimmune disease working party of the EBMT (European Group for Blood and Marrow Transplantation). Hematopoietic stem cell transplantation for multiple sclerosis: a retrospective multicenter study. J Neurol. 2002;249(8):1088-97.

29. Burt RK, Cohen BA, Russell E, et al. Hematopoietic stem cell transplantation for progressive multiple sclerosis: failure of a total body irradiation-based conditioning regimen to prevent disease progression in patients with high disability scores. Blood. 2003; 102(7):2373-8.

30. Voltarelli JC. Transplante de células-tronco hematopoéticas no diabete melito do tipo I. Rev. Bras. Hematol. Hemoter. 2004;26 (1):43-5.

31. Burt RK, Traynor A, Statkute L, et al. Nonmyeloablative hematopoietic stem cell transplantation for systemic lupus erythematosus. JAMA. 2006;295(5):527-35.

32. Jayne D, Passweg J, Marmont A, et al; European Group for Blood and Marrow Transplantation, European League Against Rheumatism Registry. Autologous stem cell transplantation for systemic lupus erythematosus. Lupus. 2004;13(3):168-76.

33. Saccardi R, Kozak T, Bocelli-Tyndall C, et al. Autoimmune diseases working party of EBMT. Autologous stem cell transplantation for progressive multiple sclerosis: update of the European Group for 
Blood and Marrow Transplantation autoimmune diseases working party database. Mult Scler. 2006;12(6):814-23.

34. Voltarelli JC, Oliveira MC, Stracieri AB, et al. Haematopoietic stem cell transplantation for refractory Takayasus arteritis. Rheumatology (Oxford). 2004;43(10):1308-9.

35. Voltarelli JC, Couri CE, Stracieri AB, et al. Autologous nonmyeloablative hematopoietic stem cell transplantation in newly diagnosed type 1 diabetes mellitus. JAMA. 2007;297(14):1568-76.

36. Torrente-Segarra V, Lisbona-Pérez M, Rotes-Sala D, Castro-Oreiro S, Carbonell-Abelió J. Clinical biological and ultrasonographic remission in a patient with muskolectal systemic lupus erythematosus with rituximab. Lupus. 2009;18(3):270-2 .

37. Robak E, Robak T. Monoclonal antibodies in the treatment of systemic lupus erythematosus. Curr Drug Targets. 2009;10 (1):26-37.

38. Garcia-Carrasco M, Jimenez-Hernandez M, Escarcega RO, MendozaPinto C, Galarza-Maldonado C, et al. Use of rituximab in patients with systemic lupus erythematosus: an update. Autoimmun rev 2009; 8(4)343-348.

39. Loherer PJ, Lauer R, Roth BJ, Wiliams SD, Kalasinsky LA, et al. Salvage therapy in recurrent germ cell cancer; ifosfamide and cisplatin plus either vinblastine or etoposide. Ann Int Med. 1988; 109:540-6.

40. Pico JL, Ostronoff JP, Droz D, et al. High-dose chemotherapy with cisplatin, etoposide and cyclofosfamide (PEC protocol) followed by autologous bone marrow suport in non seminomatous germ cell tumour. Proc Am Soc Clin Oncol. 1989;8:12 abstract 41

41. Peter W, Ross M, Vredenburgh J, Meisenberg B, Marks LB, et al. High-dose chemotherapy and autologous bone marrow support as consolidation after standard - dose adjuvant therapy for high-risk primary breast cancer. J Clin Oncol. 1993;11:1132-43.

42. Bezwoda WR, Seymour L, Dansey R. High-dose chemotherapy with hematopoietic rescue as primary treatment for metastatic breast cancer: a randomized trial. J Clin Oncol. 1995;13(10): 2483-9.

43. Bezwoda WR. Randomised controlled trial of high-dose chemotherapy versus standard dose chemotherapy for high-risk, surgically treated, primary breast cancer. Proc Am Soc Clin Oncoil 1998;18:2a

44. Weiss RB, Rifkin M, Steward FM, Thierialt RL Williams LA, et al. High-dose chemoterapy for high-risk primary breast cancer. Lancet 2000;355:999-1003.

45. Antman K. Randomized trials of high-dose chemotherapy in breast cancer: fraud, the press and the data (or lessons learned in medical policy governing clinical research). Trans Am Clin Climatol Assoc. 2002;113:56-66.

46. Bregni M. HSCT for solid tumours in adults cap 31: $464-471$, in Apperley J, Carreras E, Gluckman E, Gratwohl A. e Masszi T. The EBMT Hand Book - Haematopoietic Stem Cell TransplantationEBMT/ESH, Forum Service Editore Itália $-5^{\text {a }}$ ed. 2008, 592 p.

47. Ljungman P, Gratwöhl A, Indications and current practice for allogeneic and autologous Hsct for haematological diseases, solid tumours and immune diseases. cap. 17: 319-326 in Apperley J, Carreras E, Gluckman E, Gratwohl A e Masszi T. The EBMT Hand Book - haematopoietic Stem Cell Transplantation- EBMT/ESH, Forum Service Editore Itália $-5^{\text {a }}$ ed. 2008, 592 p.

48. Ueno NT, Rizzo JD, Demirer T, Chen YC, Hegenbart U et al. Allogeneic hematopoietic cell transplantation for metastatic breast cancer. Bone Marrow Transplant 2008;41(6):537-45

49. Yalamanchili K, Lalmuanpuii J, Wahdeed F, Farjami S, Kancherla $\mathrm{R}$, et al. High-dose chemotherapy with autologous stem cell rescue in stage IIB inflamatory. Anticancer Res. 2008;28(5B): 3139-42.
50. Demirer T, Barkholt L, Blaise D, Pedrazzoli P, Aglietta M, et al. Transplantation of allogeneic hematopoietic stem cells: treatment modality for solid tumours. Nat Clin Pract Oncol 2008;5(5):256-67.

51. Bregni M, Ueno NT, Childs R, et al. The second international meeting on allogeneic transplantation in solid tumours. Bone Marrow Transplant. 2006;38(8):527-37.

52. De Giorgi U, Blaise D, Langue A, Viens P, Marangolo M, Madroszyk $\mathrm{D}$, et al. High-dose chemotherapy with peripheral blood progenitor cell suport for patients with no-small cell lung cancer: the experience of the European Group for bone marrow transplantation (EBMT) Solid Tumours Working Party. Bone Marrow Transplant. 2007;40(11):1045-8.

53. Massenkeil, Roigas, Nagy, Wille A, Stroszcznski C, et al. Nonmyeloablative in metastatic renal cell carcinoma: delayed graftversus tumour effect is associated with chimerism conversion but transplantation has high toxicity. Bone Marrow Transplant. 2004;34:309-16

54. Abrey LE, Childs BH, Paleologos N, Kaminer L, Rosenfeld S, Salzman D, et al. High-dose chemotherapy with stem cell rescue as initial therapy for anaplastic oligodendroglioma: long-term follow-up. Neuro Oncol. 2006;8(2):183-8.

55. Ballen KK, Colvin G, Dey BR, Porter D, Westerwelt P, et al. Cellular immune therapy for refractory cancers: as novel therapy strategies. Exp Hematol. 2005;33(12):1427-35.

Avaliação: O tema apresentado consta da pauta elaborada pelo editor, Professor Milton Artur Ruiz, e coeditores deste suplemento, Professores Sergio Paulo Bydlowski e Adriana Seber.

Conflito de interesse: não declarado

Recebido: 24/03/2009

Aceito: 31/03/2009 